(Aus der kgl. ung. thierphysiol. Versuchsstation in Budapest.)

\title{
Zur Kenntniss des P-, Ca- und Mg-Umsatzes bei Pflanzenfressern.
}

Von

\author{
Prof. Dr. med. F. Tangl.
}

Gelegentlich der Fütterungsversuche, die mit Rieselwiesenheu an Pferden ausgeführt wurden, ergab die Aschenanalyse, dass das Heu ausserordentlich arm an Kalk war. Dies veranlasste mich, in zwei Versuchsreihen den Ca-Umsatz und zugleich den P- und:Mg-Umsatz bei zwei Pferden (Nr. III und IV, gesunde kräftige 10- resp. 14 jährige Wallachen) zu bestimmen, um so mehr, als meines Wissens bisher nur sehr wenige Untersuchungen über den anorganischen Stoffwechsel bei Pferden, überhaupt bei Pflanzenfressern, vorliegen und man über das Minimum, welches erwachsene Thiere an den erwähnten Elementen benöthigen, noch gar nichts weiss. So weit ich aus der mir zugänglichen Literatur ersehe, hat sich mit dem anorganischen Stoffwechsel des erwachsenen Pferdes nur E. Wolff ${ }^{1}$ ) beschäftigt. Er hat bei ein em Pferde zu verschiedenen Zeiten und bei verschiedener Fütterung den gesammten anorganischen Stoffwechsel bestimmt. Wenn auch gegen die Exactheit der Wolff'schen Versuche kein Einwand erhoben werden kann, so muss doch erwähnt werden, dass sie bezüglich des P-Stoffwechsels insofern mangelhaft sind, als mit Ausnahme einer einzigen Versuchsperiode der P.Gehalt des Harnes nicht bestimmt wurde, wohl in der Voraussetzung, dass dieser wegen seiner geringen Grösse vernachlässigt werden kann.

Leider erlaubten es äussere Umstände nicht, dass ich meine Untersuchungen auf den gesammten anorganischen Stoffwechsel ausdehne; ich beschränkte mich also auf die Bestimmung des P-, Ca-

1) E. Wolff, Grundlage für die rationelle Fütterung des Pferdes S. 53. Berlin 1886 und Grundlage für die rationelle Fütterung des Pferdes. Neue Beiträge S. 36. Berlin 1887. 
und Mg-Stoffwechsels. Bezüglich des P-Stoffwechsels ergänzen meine Versuche gewissermaassen diejenigen von Wolff, da ich auch den P-Gehalt des Harnes stets bestimmt habe.

Die Versuchsreihen, bei welchen diese Untersuchungen ausgeführt wurden, dienten, wie bereits erwähnt, dazu, die Bekömmlichkeit und Verdaulichkeit des Rieselwiesenheues zu bestimmen. Die Versuchsanordnung, die übrigens an anderer Stelle - (Die landwirthschaftl. Versuchsstationen Bd. 56), - wo diese Ausnützungsversuche publicirt werden, ausführlich besprochen ist, war in der üblichen Weise derart getroffen, dass sowohl die Einnahmen als die in Betracht kommenden Ausgaben genau gesammelt und analysirt werdeı konnten. Es genügt also, wenn ich hier diesbezüglich sowie bezüglich der übriqen Einzelheiten des Versuches und der analytischen Daten auf jene Mittheilung verweise und hier nur dasjenige anführe, was zum P-, Ca- und Mg-Stoffwechsel in Beziehung steht resp. zur Beurtheilung der diesbezüglichen Resultate nothwendig ist.

Was zunächst die angewendeten analytischen Methoden betrifft, so wurden bei den $\mathrm{Ca}$, $\mathrm{Mg}$ - und P-Bestimmungen im Grossen und Ganzen die allgemein bekannten und verlässlichsten Methoden verwendet. Die Ca-Bestimmungen wurden in der Weise ausgeführt, dass das beim Veraschen durch die $\mathrm{SiO}_{2}$ eventuell gebundene $\mathrm{Ca}$ nicht verloren gehe. Die aus 5-8 g Substanz - Futter oder lufttrockener Koth - gewonnene Rohasche wurde mit der doppelten Menge vorher ausgeglühten chemisch reinen, $\mathrm{Na}_{2} \mathrm{CO}_{3}$ gut vermengt und dann vorsichtig geschmolzen. Die abgekühlte Masse wurde in verdünnter $\mathrm{HCl}$ gelöst, eingedampft, dann $1 / 2$ Stunde bei $110^{\circ} \mathrm{C}$. im Trockenschrank getrocknet. Mit Ausnahme der frei und unlöslich gemachten $\mathrm{SiO}_{2}$ löst sich dann alles in verdunnter $\mathrm{HCl}$. Aus der filtrirten Lösung, die nunmehr sämmtliches $\mathrm{Ca}$ enthielt, wurde nach erfolgter Neutralisation mit $\mathrm{NH}_{3}$ das $\mathrm{Ca}$ in der üblichen Weise mit Ammoniumoxalat gefällt, geglüht und als $\mathrm{CaO}$ gewogen. Aus der vom $\mathrm{Ca}(\mathrm{COO})_{2}$-Niederschlag ahfiltrirten, mit $\mathrm{NH}_{3}$ versetzten Lösung wurde das $\mathrm{Mg}$ mit $\mathrm{Na}_{2} \mathrm{HPO}_{4}$ gefällt und als $\mathrm{Mg}_{2} \mathrm{P}_{2} \mathrm{O}_{7}$ gewogen. Aehnlich verfuhren wir bei der Bestimmung des $\mathrm{Ca}$ und $\mathrm{Mg}$ im Harne, von dem $50 \mathrm{cem}$ zu je einer Analyse genommen wurden.

Zur P-Bestimmung wurden statt der Veraschung $5-8 \mathrm{~g}$ lufttrockenen Kothes resp. Futters, 40 ecm concentrirter $\mathrm{H}_{2} \mathrm{SO}_{4}$ in Kjeldahl-Kolben ganz so wie bei der N-Bestimmung aufgesehlossen. Nach vollendeter Oxydation wurde der Kolbeninhalt mit Wasser ver- 
dünnt, und die ausgeschiedene $\mathrm{SiO}_{2}$ durch Filtriren entfernt und im Filtrat die Phosphorsäure mittelst der Molybdänmethode bestimmt. Der Harn $(500 \mathrm{ccm})$ wurde statt des Veraschens mit $\mathrm{HNO}_{3}$ gekocht und dann die Phosphorsäure mit Ammoniummolybdat gefällt.

Da die Thiere ausser dem Futter auch mit dem Tränkwasser $\mathrm{Ca}$ und $\mathrm{Mg}$ aufgenommen haben, so wurden auch im Wasser (1000 cem) $\mathrm{Ca}$ und $\mathrm{Mg}$ bestimmt.

Sämmtliche Analysen wurden doppelt ausgeführt, die mitgetheilten Werthe sind Mittelwerthe von wenigstens zwei gut übereinstimmenden Analysen. Wie aus den weiter unten angefübrten Tabellen ersichtlich, habe ich die Resultate der Analysen nicht in der noch immer tublichen Form als $\mathrm{CaO}$ resp. $\mathrm{MgO}$ und $\mathrm{P}_{2} \mathrm{O}_{5}$ ausgedrückt, welche ${ }_{n}$ ultraconservativen" Formen - wie Ostwald sie nennt - dem heutigen Stande der wissenschaftlichen Chemie nicht mehr entsprechen. Als die einfachste und beste Art, die Ergebnisse der Analyse darzustellen, bezeichnet $O$ stwald "die Aufführung der einzelnen Elemente mit den Mengen, in denen sie vorhanden sind" 1 ). Den Vorschlag Ostwald's befolgend führte ich die Rechnung auf die Elemente selbst, bezeichne also deren Menge als Ca resp. Mg und P. Es wäre sehr wünschenswerth, dass in allen rein wissenschaftlichen Arbeiten diese einfachste und rationelle Form Anwendung fände.

Ausser den angeführten Stoffen wurde in den Einnahmen und Ausgaben auch der $\mathrm{N}$ (nach Kjeldahl) bestimmt, also auch der $\mathrm{N}$-Stoffwechsel ermittelt.

Im Ganzen wurde der N-, P-, Ca- und Mg-Umsatz in zwei Versuchsreihen bei zwei Pferden Nr. III und IV bestimmt. In der 1. Versuchsreihe, die acht Tage, vom 2. März bis 9. März währte, erhielten die Thiere als Futter $8 \mathrm{~kg} \mathrm{Heu}$; in der 2. Versuchsreihe, die vom 15. - 20. April, also sechs Tage dauerte, war das Futter $4 \mathrm{~kg}$ Hafer und $5 \mathrm{~kg}$ Heu. Im täglichen Harn wurde nur der $\mathrm{N}$ bestimmt; zur $\mathrm{P}-, \mathrm{Ca}$ - und $\mathrm{Mg}$-Bestimmung wurde fast immer der Harn von je zwei Tagen vermischt. Vom täglichen Kothe wurde je $1 \%$ genau abgewogen, bei $60^{\circ}$ getrocknet, zwei bis drei Tage im Zimmer stehen gelassen, gewogen, und dann vermischt und fein gemablen, und dieses lufttrockene Pulver analysirt.

1) Ostwald, Die wissenschaftlichen Grundlagen der analyt. Chemie, 2. Aufl., S. 197.1897. 


\section{Versuchsreihe 1.}

Futter: $8 \mathrm{~kg}$ Heu. Das Hen enthielt.

$$
\begin{array}{llll}
\mathrm{N} & 1,366 \% & \mathrm{Ca} & 0,440 \% \\
\mathrm{P} & 0,146 \% & \mathrm{Mg} & 0,137 \% .
\end{array}
$$

Das Tränkwasser enthielt $0,0049 \% \mathrm{Ca}$ und $0,0013 \mathrm{~g} \mathrm{Mg}$.

$\begin{array}{ccc} & \text { Pferd III } & \text { Pferd IV } \\ \text { Körpergewicht am Anfange des Versuchs } & 408 \mathrm{~kg} & 445 \mathrm{~kg} \\ " \text { Ende " } & 404 " & 440 " \\ \text { Durchschnittliches Körpergewicht ') } & 406 " & 442 " \\ \text { Tränkwasser pro Tag (durchschnittlich) } & 19,6, & 26,5 "\end{array}$

Menge und Gehalt des Harns und Koths an N, P, Ca und Mg zeigt Tabelle 1 auf S. 231.

\section{Versuchsreihe 2.}

Futter: $4 \mathrm{~kg}$ Heu +5 kg Hafer.

$\begin{array}{ccc} & \text { Der Hafer } & \text { Das Heu enthielt: } \\ \mathrm{N} & 2,272 \% & \mathbf{1 , 8 4 4 \%} \\ \mathrm{P} & 0,406 \% & 0,165 \% \\ \mathrm{Ca} & 0,143 \% & 0,410 \% \\ \mathrm{Mg} & 0,104 \% & 0,209 \%\end{array}$

\begin{tabular}{|c|c|c|}
\hline & Pferd III & Pferd IV \\
\hline Körpergewicht am Anfange des Versuchs & $403,8 \mathrm{~kg}$ & $432,5 \mathrm{~kg}$ \\
\hline 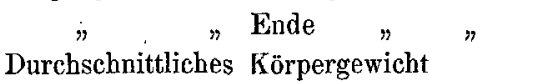 & $\begin{array}{l}406,5 \\
405\end{array}$ & $\begin{array}{l}436,8 \eta \\
435\end{array}$ \\
\hline Tränkwasser pro Tag (durchschnittlich) & 17,3 & 22,0 \\
\hline
\end{tabular}

Im Tränkwasser waren $0,0049 \% \mathrm{Ca}$ und $0,0013 \% \mathrm{Mg}$.

In der Versuchsreihe 1 war, wie aus den angeführten Daten ersichtlich, das Futter nicht ausreichend, das Körpergewicht der Pferde nahm durchschnittlich täglich um 0,50 resp. $0,63 \mathrm{~kg} \mathrm{ab}$, während in der Versuchsreihe 2, in welcher neben dem Heu auch Hafer verabreicht wurde, das Körpergewicht täglich um 0,45 bezw. $0,72 \mathrm{~kg}$ zunahm. In letzterer Versuchsreihe nahmen die Thiere auch eine viel grössere Menge verdaulicher Nährstoffe auf, wie es aus der auf S. 232 folgenden Tabelle hervorgeht.

1) Mittel aus den täglichen Wägungen. 
Zur Kenntniss des P-, Ca- und Mg-Umsatzes bei Pflanzenfressern.

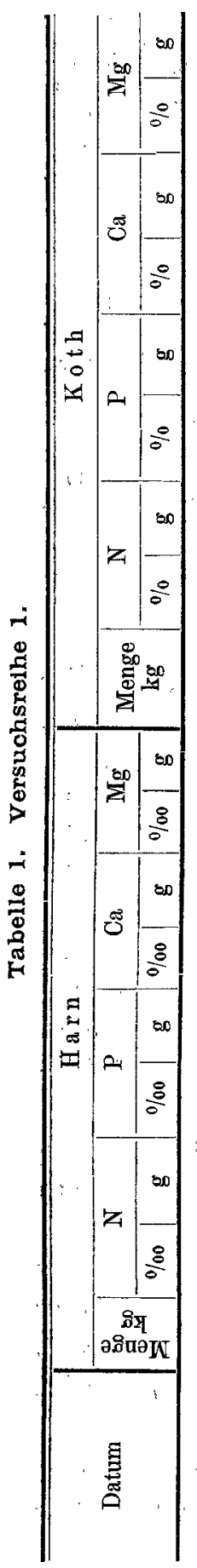

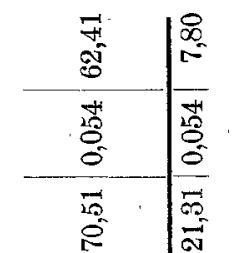
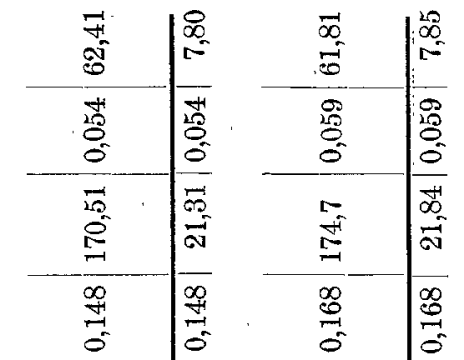

要

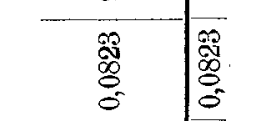

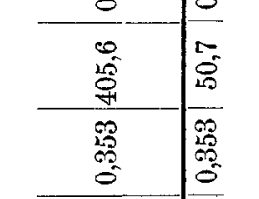

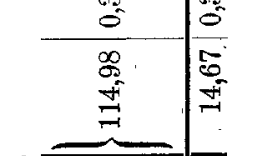

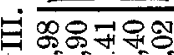

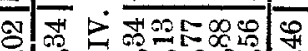

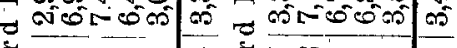

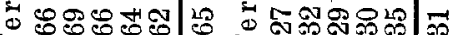

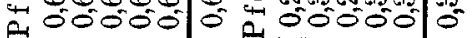

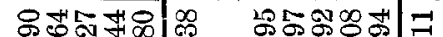

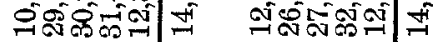
Q कीजिएक की

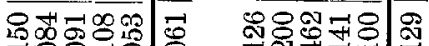
700 00000

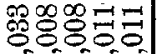
00000 군으용요 8 年 䒠牙思

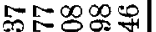
किजी

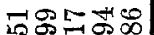
संजिले

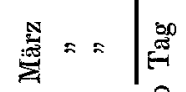

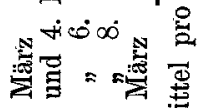

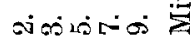

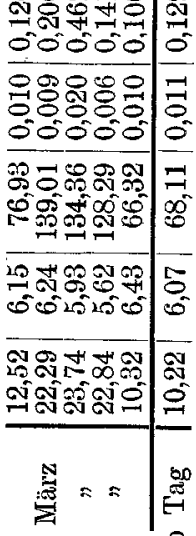

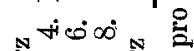

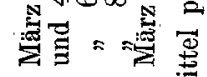
बisisios

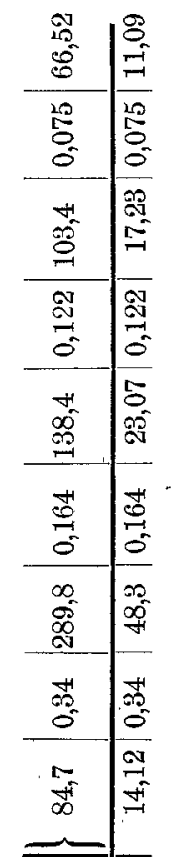

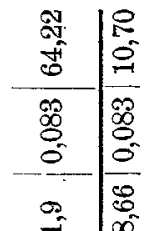

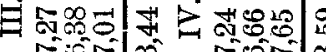

क क 00 क ¿ A 0000000 ก 푸응 की किष्ठ (1)

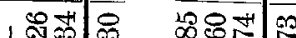
-7)

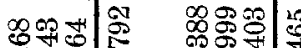

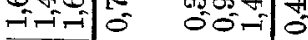
12:日十

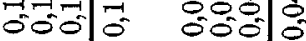
or- ov $\quad 0 \pi 0$

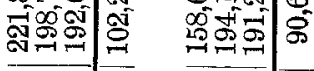

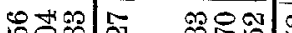

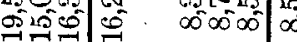
सम Fis

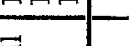
荡== ن용 $\overrightarrow{\mathrm{g}}==\overrightarrow{\mathrm{B}}$

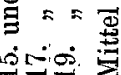
क्नेत्री 荇: = $\dot{\infty} \dot{\infty}$ $\bar{\Xi}==\vec{\Phi}$

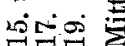




\begin{tabular}{|c|c|c|c|c|c|}
\hline 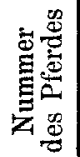 & $\begin{array}{c}\text { Organ. } \\
\text { Sub- } \\
\text { stanz } \\
\mathrm{g}\end{array}$ & $\left|\begin{array}{c}\text { Roh- } \\
\text { proteïn } \\
(\mathrm{N} \times 6,25) \\
\mathrm{g}\end{array}\right|$ & $\begin{array}{c}\text { Fett } \\
\text { (A ether- } \\
\text { extract) } \\
\mathrm{g}\end{array}$ & $\begin{array}{c}\text { Roh- } \\
\text { faser } \\
\mathrm{g}\end{array}$ & $\begin{array}{c}\text { N-freie } \\
\text { Extract } \\
\text { stoffe } \\
\text { g }\end{array}$ \\
\hline
\end{tabular}

Versuchsreihe 1 .

\begin{tabular}{|c|c|c|c|c|c|}
\hline $\begin{array}{l}\text { Mit dem Futter wurden täg- } \\
\text { lich aufgenommen } \\
\text { Mit dem Kothe entleert }\end{array}$ & $\begin{array}{l}6084 \\
3133\end{array}$ & $\begin{array}{l}683 \\
317\end{array}$ & $\begin{array}{l}153 \\
137\end{array}$ & $\begin{array}{l}2482 \\
1147\end{array}$ & $\begin{array}{l}2766 \\
1533\end{array}$ \\
\hline Also resorbirt. . . . & $\begin{array}{l}2951 \\
-48,5 \%\end{array}$ & 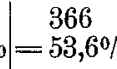 & $\begin{array}{c}16 \\
=10,8 \%\end{array}$ & $\begin{array}{c}1335 \\
-53,8 \%\end{array}$ & $\begin{array}{l}1233 \\
=44,6 \%\end{array}$ \\
\hline $\begin{array}{l}\text { Mit dem Futter wurden täg- } \\
\text { lich aufgenommen } \\
\text { Mit dem Kothe entleert }\end{array}$ & $\begin{array}{l}6084 \\
2896 \\
\end{array}$ & $\begin{array}{l}683 \\
331 \\
\end{array}$ & $\begin{array}{l}153 \\
130 \\
\end{array}$ & $\begin{array}{l}2482 \\
1138 \\
\end{array}$ & $\begin{array}{l}2766 \\
1297 \\
\end{array}$ \\
\hline Also resorbirt. . . . & $\begin{array}{r}3188 \\
=52,4\end{array}$ & 352 & 28 & $\begin{array}{r}1344 \\
=54,2\end{array}$ & $\begin{array}{c}1469 \\
=53,2 \%\end{array}$ \\
\hline
\end{tabular}

Versuchsreihe 2.

\begin{tabular}{|c|c|c|c|c|c|}
\hline $\begin{array}{l}\text { Mit dem Futter wurden täg- } \\
\text { lich aufgenommen . } \\
\text { Mit dem Kothe entleert }\end{array}$ & $\begin{array}{l}7417 \\
3355 \\
\end{array}$ & $\begin{array}{r}1144 \\
302 \\
\end{array}$ & $\begin{array}{l}351 \\
158 \\
\end{array}$ & $\begin{array}{l}1771 \\
1264 \\
\end{array}$ & $\begin{array}{l}4151 \\
1631 \\
\end{array}$ \\
\hline Also resorbirt. . . . & $\begin{aligned} & 4062 \\
= & 54,8 \%\end{aligned}$ & $\begin{array}{r}842 \\
-73,6^{0} \%\end{array}$ & $\begin{array}{c}193 \\
=55,2 \%\end{array}$ & $\begin{array}{c}507 \\
=28,6 \%\end{array}$ & $\begin{array}{c}2520 \\
=63,6^{\circ} \%\end{array}$ \\
\hline $\begin{array}{l}\text { Mit dem Futter wurden täg- } \\
\text { lich aufgenommen . } \\
\text { Mit dem Kothe entleert }\end{array}$ & $\begin{array}{l}7417 \\
3200\end{array}$ & $\begin{array}{r}1144 \\
342\end{array}$ & $\begin{array}{l}351 \\
142\end{array}$ & $\begin{array}{l}1771 \\
1156\end{array}$ & $\begin{array}{l}4151 \\
1560\end{array}$ \\
\hline Also resorbirt. & 4217 & 8 & $\begin{aligned} & 209 \\
= & 59,4^{\circ}\end{aligned}$ & $\begin{array}{c}615 \\
=34,7 \%\end{array}$ & 25 \\
\hline
\end{tabular}

Dass das Futter in der 1. Versuchsreihe thatsächlich ungenügend war, bewies nicht nur die Abnahme des Körpergewichtes, sondern, wie später dargethan werden wird, auch der N-Stoffwechsel; aus letzterem ging auch hervor, dass in der 2. Versuchsreihe ein Fleischansatz stattgefunden hat.

Versuchsreihe 1 bot lalso die Gelegenheit, den P-, Ca- und MgStoffwechsel bei ungenügender, die zweite bei zur Production von Körpersubstanz ausreichendem Futter zu untersuchen.

Was den Gehalt des Futters an $\mathrm{P}, \mathrm{Ca}$ und $\mathrm{Mg}$ betrifft, so fällt vor Allem die grosse Kalkarmuth des Heues und dessen sehr hoher P-Gehalt auf, während der Mg-Gehalt nichts Auffälliges aufweist. Der Hafer enthält relativ viel $\mathrm{Ca}$ und auffallend viel $\mathrm{P}$. Um meine Daten augenscheinlicher mit den Daten E. Wolff's vergleichen zu können, habe ich sie auf $\mathrm{P}_{2} \mathrm{O}_{5}, \mathrm{CaO}$ und $\mathrm{MgO}$ umgerechnet und in der folgenden Zusammensetzung neben die von E. Wolff berechneten Mittelwerthe gestellt: 
Zur Kenntniss des P-, Ca- und Mg-Umsatzes bei Pflanzenfressern.

$100 \mathrm{~g}$ Trockensubstanz enthalten

$\begin{array}{ccc}\mathrm{CaO} & \mathrm{MgO} & \mathrm{P}_{2} \mathrm{O}_{5} \\ \mathrm{~g} & \mathrm{~g} & \mathrm{~g}\end{array}$

Heu der Versuchsreihe $1 \ldots$. . . $0,794 \quad 0,273 \quad 0,804$

Heu der Versuchsreihe 2 . . . $0,654 \quad 0,394 \quad 0,860$

Nach E. Wolff ${ }^{1}$ ). Wiesenheu:

Heu von Wasserwiesen . . . . . $1,113 \quad 0,4810.496$

Ungesundes Heu ${ }^{2}$ ) . . . . . . . $0,0,629 \quad 0,312 \quad 0,263$

(Maxim. 1,30 0,525)

Hafer unserer Versuche. . . . . $0,232 \quad 0,251 \quad 2,15$

Hafer, Mittel nach E. Wolff . . $0,112 \quad 0,223 \quad 0,80$

(Maxim. 0,361 1,509)

Morgen ${ }^{3}$ ) fand in zwei Proben von Wiesenheu, bei dessen Verfütterung Kochenbrüchigkeit auftrat, in $100 \mathrm{~g}$ Trockensubstanz 0,44 resp. $0,79 \mathrm{~g} \mathrm{CaO}$ und 0,24 resp. $0,31 \mathrm{~g} \mathrm{P}_{2} \mathrm{O}_{5}$.

Es ist also zweifellos, dass das Heu in meinen Versuchen sehr kalkarm war, und zwar so kalkarm wie das ungesunde Heu aus Gegenden, in welchen Knochenbrüchigkeit beim Rinde häufig ist. Dagegen enthielt es aber sehr viel $P$, mehr als nach $W$ olf f durchschnittlich gutes Wiesenheu. Auch der Hafer enthielt bedeutend mehr P. Wenn auch dieser Unterschied theilweise in der Verschiedenheit der angewandten analytisehen Methode seine Erklärung finden könnte, so ist er doch zu bedeutend, um nur ausschliesslich darauf zurückgeführt werden zu können.

Da der Hafer weniger Ca enthält als das Heu und bedeutend mehr P, so verzehrten die Pferde in der 1 . Versuchsreihe bedeutend mehr $\mathrm{Ca}$ und weniger $\mathrm{P}$ als in der 2. Versuchsreihe; beim $\mathrm{Mg}$ war der Unterschied nur ein geringer. Es wurden nämlich verzehrt in der

\begin{tabular}{cccc}
\multicolumn{4}{c}{ Versuchsreihe 1} \\
& $\mathrm{P}$ & $\mathrm{Ca}$ & $\mathrm{Mg}$ \\
$\mathrm{g}$ & $\mathrm{g}$ & $\mathrm{g}$ \\
von Pferd III & 11,74 & $\left.36,38^{4}\right)$ & $\left.11,23^{4}\right)$ \\
von Pferd IV & 11,74 & 36,72 & 11,33 \\
Versuchsreihe 2 \\
von Pferd III & 24,50 & $\left.27,23^{4}\right)$ & $\left.14,86^{4}\right)$ \\
von Pferd IV & 24,50 & 27,44 & 14,92
\end{tabular}

1) E. Wolff, Aschenanalysen landw. u. forstw. Prod. II. Theil S. 141.

2) Aus Gegenden, wo die Knochenbrüchigkeit der Thiere häufig vorkommt.

3) Morgen, Die landwirthsch. Versuchsstationen Bd. 23 S. 442.

4) $\mathrm{Da}$ ist das mit dem Tränkwasser aufgenommene $\mathrm{Ca}$ und $\mathrm{Mg}$ schon eingerechnet; das bedingt den kleinen Unterschied in der Ca- und Mg-Aufnahme der zwei Pferde. 
Was die Ausnützung dieser Stoffe betrifft, so darf nicht vergessen werden, dass, wie zuerst $V_{0}$ it nachgewiesen hat, die Darmschleimhaut ebenso wie die. Nieren Ca- und Mg.Phosphat aus dem Blute ausscheidet ${ }_{1}^{1}$ ), ja, dass der Darm, wie Noorden ${ }^{2}$ ) sagt, die eigentliche Secretionsstätte für den Kalk ist. Der Ca-, Mg- und PGehalt des Kothes ist also kein Maass für die Verdaulichkeit dieser Stoffe.

In der Versuchsreihe haben die Thiere mit dem Kothe ebenso viel $\mathrm{P}$ entleert, als sie mit dem Futter aufgenommen haben. Der minimale P-Ueberschuss im Koth liegt weit innerhalb der Grenzen der Versuchsfehler. In der 2. Versuchsreihe wurde mit dem Kothe etwas weniger $P$ entleert als mit dem Futter aufgenommen. Den Veriust an $\mathrm{P}, \mathrm{Ca}$ und $\mathrm{Mg}$ durch den Koth in Procenten der im Futter resp. Futter und Tränkwasser aufgenommenen Menge dieses Stoffes zeigen folgende Zahlen:

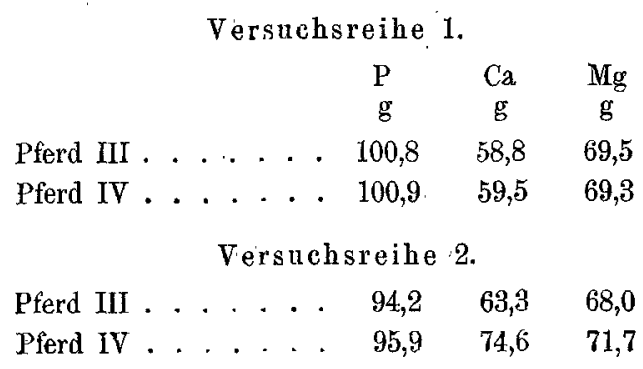

Diese Zahlen stimmen mit denjenigen $E$. $\mathrm{W}_{0} \mathrm{lff}^{\prime} \mathrm{s}_{1}{ }^{\mathrm{a}}$ ). nur theilweise überein. In E. Wolff's Versuchen gingen mit dem Kothe vom $\mathrm{Ca} 35,36-42,96 \%$, vom $\mathrm{Mg} 59,24-61,84 \%$ verloren, also weniger wie in meinen Versuchen, nach welchen mehr als $2 / 3$ des $\mathrm{Ca}$ und fast $3 / 4$ des $\mathrm{Mg}$ des Futters mit dem Kothe entleert werden können. Der P wird, wie auch E. Wolff fand, ganz oder fast ganz mit dem Kothe entleert.

In der Versuchsreihe 2, in welcher die Pferde neben Heu auch Hafer erhielten, ging mit dem Kothe relativ mehr $\mathrm{Ca}$ und $\mathrm{Mg}$ verloren, wie in der Versuchsreihe 1. Vielleicht sind das $\mathrm{Ca}$ und $\mathrm{Mg}$ im Hafer in weniger leicht verdaulicher resp. löslicher Verbindung

1) Voit in Hermann's Handb. d. Physiol. Bd. 6 S. 373.

2) Noorden, Lehrb. d. Pathologie des Stofiwechsels S. 20.

3) E. Wolff, Grundl. d. ration. Fütterung etc. Neue Beiträge 1897 S. 40. 
vorhanden oder setzt der Hafer die Verdaulichkeit der Ca-Verbindungen des Heues herab. Meine Versuche entscheiden diese Frage nicht.

Da der Gehalt des Harnes an P, Ca und $\mathrm{Mg}$ in erster Reihe von der resorbirten Menge dieser Stoffe abhängt, so war im Harn wenig $\mathrm{P}$ und relativ viel $\mathrm{Ca}$ und $\mathrm{Mg}$ zu erwarten. Im ersten Versuch fand sich auch thatsächlich im Harn beider Pferde - besonders. bei Pferd III -- nur sehr wenig P; bei letzterem Pferde nur $0,06 \mathrm{~g} \mathrm{P}$ pro Tag. doch war das Minimum $0,042 \mathrm{~g}$, so dass der Harn nur $0,0008 \% \mathrm{P}$ enthielt. Etwas mehr P entleerte das Pferd 2. Hingegen entleerten die Pferde in der 2. Versuchsreihe mit dem Harne nicht unbedeutende Mengen P, Perd III täglich $0,792 \mathrm{~g}$ und Pferd IV $0,465 \mathrm{~g}$, Mengen, die durchaus nicht $\mathrm{zu}$ vernachlässigen sind. (Ist doch $0,792 \mathrm{~g} \mathrm{P}=3,63 \mathrm{~g} \mathrm{P}_{2} \mathrm{O}_{5}$ und $0,465 \mathrm{~g} \mathrm{P}=2,13 \mathrm{~g}$ $\mathrm{P}_{2} \mathrm{O}_{5}$.) Jedenfalls beweisen diese Zahlen, dass Pferde bei P-reichem Futter - Hafer - nicht unbedeutende Mengen $P$ mit dem Harn entleeren.

E. Wolff bestimmte nur in einer Periode seiner 2. Versuchsreihe den $P$-Gehalt des Harnes und fand pro Tag 0,88 $\mathrm{g} \mathrm{P}_{2} \mathrm{O}_{5}$; mit dem Futter verzehrte das Pferd $62,79 \mathrm{~g} \mathrm{P}_{2} \mathrm{O}_{5}$; Salkow skil) fand im 24 stündigen Harn eines Pferdes $0,22 \mathrm{~g} \mathrm{P}_{2} \mathrm{O}_{5}$.

Die Menge des im Harn entleerten $\mathrm{Ca}$ und $\mathrm{Mg}$ entspricht annähernd der resorbirten Menge. Sie ist kleiner als bei $\mathrm{Ca}$ und $\mathrm{Mg}$ reichem Futter. Wenn es auch nicht zu bezweifeln ist, dass das $\mathrm{Ca}$ und $\mathrm{Mg}$ im Harn aus dem Futter stammt, ist es immerhin interessant, dass der Quotient $\frac{\mathrm{Mg}}{\mathrm{Ca}}$ des Harnes vollständig. übereinstimmt mit dem Verhältniss des resorbirten $\mathrm{Ca}$ zum resorbirten $\mathrm{Mg}$ :

Resorbirt Mit dem Harn entleert

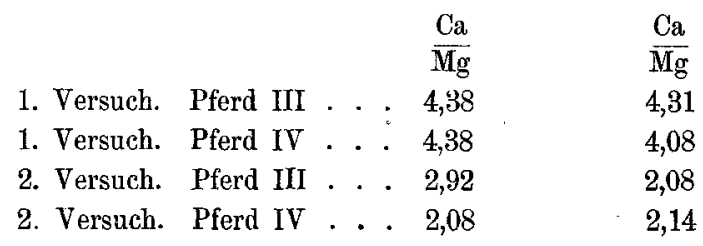

Den $\mathrm{P}$-, Ca- und Mg-Stoff weehsel pro Tag zeigt die folgende Tabelle, in die ich auch den N-Stoffwechsel aufgenommen habe.

1) Gitirt nach Tereg und Ellenberger, siehe Vergl. Physiol. der Haussäungethiere Bd. $1 \mathrm{~S} .382$. 
Tabelle 3.

Täglicher N-, P-, Ca- und Mg-Umsatz.

\begin{tabular}{l|c|c|c|c|c}
\hline & $\mathrm{N}$ & $\mathrm{P}$ & $\mathrm{Ca}$ & $\mathrm{Mg}$ \\
& $\mathrm{g}$ & $\mathrm{g}$ & $\mathrm{g}$ & $\mathrm{g}$ \\
\hline
\end{tabular}

Versuchsreihe 1.

Pferd III.

\begin{tabular}{ll|l|l|l|l|l} 
Futter: $8 \mathrm{~kg}$ Heu &. & 109,28 & 11,74 & 35,40 & 10,97
\end{tabular}

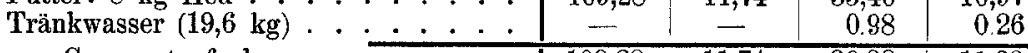

\begin{tabular}{ll|l|l|l|l|l|} 
Gesammtaufnahme . . & $\cdots$ & $\cdots$ & 109,28 & 11,74 & 36,38 & 11,23 \\
\hline
\end{tabular}

\begin{tabular}{|l|l|l|l|l|l} 
Koth. . . . . . . . . . . . . & 50,70 & 11,83 & 21,31 & 7,80 \\
\hline
\end{tabular}

\begin{tabular}{ll|l|l|l|l|l|l|} 
Also resorbirt. . . . . . . & 58,58 & $(-0,09)$ & 15,07 & 3,43
\end{tabular}

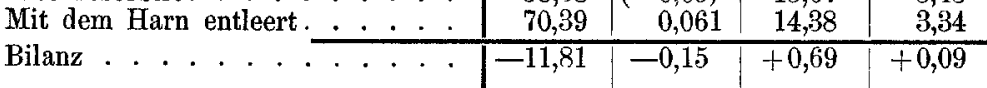

(Durchschnittliche Veränderung des Körpergewichtes pro Tag: - 0,5 kg.)

Pferd IV.

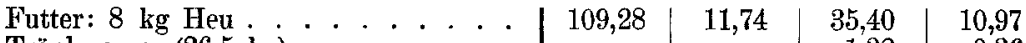

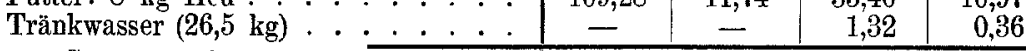

\begin{tabular}{|l|l|l|l|l|l}
\hline Gesammtaufnahme . . . . . . & 109,28 & 11,74 & 36,72 & 11,33
\end{tabular}

\begin{tabular}{rl|l|r|r|r} 
Koth. . . . . . . . . . . . . & 52,91 & 11,85 & 21,84 & 7,85 \\
\hline
\end{tabular}

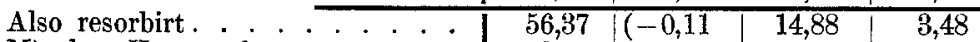

\begin{tabular}{l|r|r|r|r} 
Mit dem Harn entleert. . . . . . & 68,11 & 0,13 & 14,11 & $\mathbf{3}, 46$ \\
\hline
\end{tabular}

Bilanz . . . . . . . . . $\mid$\begin{tabular}{l|l|l|l|l|}
\hline$-11,74$ & $-0,24$ & $+0,77$ & $+0,02$
\end{tabular}

(Durchschnittliche Veränderung des Körpergewichtes pro Tag: $-0,63 \mathrm{~kg}$.)

Versuchsreihe 2 .

Pferd III.

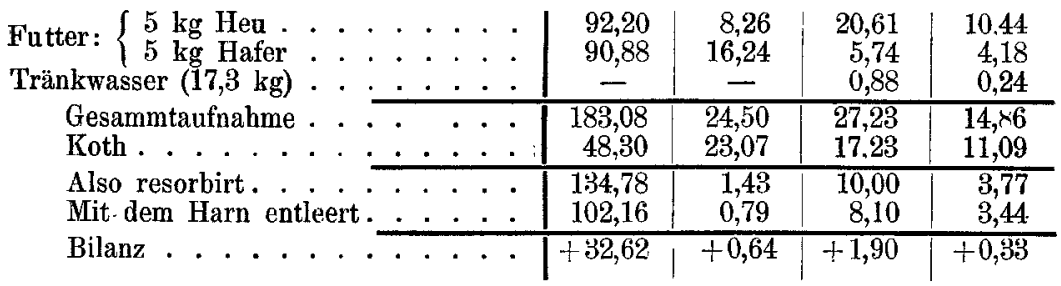

(Durchschnittliche Veränderung des Körpergewichtes pro Tag: $+0,45 \mathrm{~kg}$.)

Pferd IV.

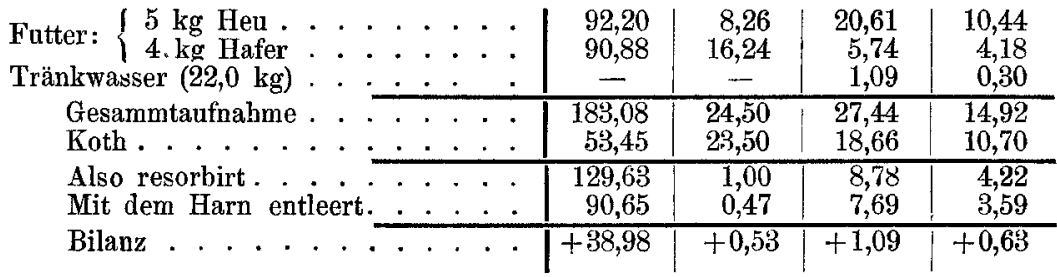

(Durchschnittliche Veränderung des Körpergewichtes pro Tag: + 0,72 kg.)

Aus dieser Tabelle geht vor Allem hervor, dass der Stoffwechsel in je einer Versuchsreihe also unter ganz. gleichen Versuchs- 
bedingungen bei beiden Pferden ganz gleich verlaufen ist, was die Zulässigkeit der aus den Ergebnissen gezogenen Schlüsse in hohem Maasse stützt.

Der N-St offwechsel beweist, dass in der ersten Versuchsreihe bei ungenügendem Futter die Thiere aus ihrem eigenen Eiweissbestande zugesetzt haben. Das thatsächliche N-Deficit ist jedenfalls grösser gewesen als das aus den Zahlen der Tabelle berechnete. Denn erstens geht beim Trocknen des Kothes immer etwas $\mathrm{N}$ verloren und zweitens verlieren die Thiere durch Ausfall von Haaren, Abnützung der Hufe, Abfall der Epidermisschuppen u. s. w. fortwährend $N$, welcher Verlust nach E. Wolff pro Tag etwa $7 g^{1}$ ) beträgt. Wir müssen das berechnete N-Deficit in unserem Versuche dementsprechend um $7 \mathrm{~g}$ erhöhen. Es hat also jedes Pferd täglich etwa $19 \mathrm{~g} \mathrm{~N}$ verloren, was annähernd $600 \mathrm{~g}$ Fleisch entspricht. (Das eine Pferd verlor täglich durchschnittlich $630 \mathrm{~g}$, das andere $500 \mathrm{~g}$ an Körpergewicht.)

Ebenso wie die Thiere sich in N-Deficit befanden, waren sie auch in P-Deficit. Es wurde schon erwähnt, dass der ganze $\mathrm{P}$ des Futters in Kothe wiedergefunden wurde, folglich kann der $\mathrm{P}$ des Harnes nur aus der Körpersubstanz stammen. Das folgt übrigens schon aus dem N-Umsatz. Dem $19 \mathrm{~g} \mathrm{~N}$-Verlust entspricht ein Fleischverlust von etwa $600 \mathrm{~g}$; wird nun so viel Fleisch zersetzt und die Zersetzungsproducte entleert, so muss natürlich auch der $\mathrm{P}$ des Fleisches in den Harn gelangen. Nach $Z$ uel ze ${ }^{2}$ ) entfallen im Pferdemuskel auf $100 \mathrm{~g} \mathrm{~N} 15,7 \mathrm{~g} \mathrm{P}_{5} \mathrm{O}_{2}$, was $3,43 \mathrm{~g} \mathrm{P}$ entspricht. Mithin würde einem N-Verlust von $19 \mathrm{~g}$ ein Verlust von $0,65 \mathrm{~g}$ $\mathrm{P}$ entsprechen; der nachgewiesene P-Verlust beträgt aber nur $0,15 \mathrm{~g}$ bei dem einen und $0,24 \mathrm{~g}$ bei dem anderen Pferde. Der beobachtete P-Verlust könnte also jedenfalls in dem aus dem N-Deficit in der üblichen Weise berechneten Fleischverlust seine Erklärung finden. Der Unterschied zwischen dem aus dem Fleischverlust berechneten und dem thatsächlich ermittelten P-Deficit ist jedoch zu bedeutend, als dass er vielleicht auf Versuchsfehler zurückgeführt werden könnte.

Die wahrscheinlichste Erklärung dieses grossen Unterschiedes ist die, dass bei ungenügender Fiweisszufuhr ausser Muskelsubstanz auch noch andere P-ärmere Gewebe zersetzt werden.

1) E. Wolff, Grundl. d. ration. Fütterung d. Pferdes. Neue Beiträge S. 106.

2) Znelzer, Untersuch. üb. die Semiologie des Harns S. 47. Berlin 1884. 
In der zweiten Versuchsreihe weist der $\mathrm{N}$-Stoffwechsel einen Eiweissansatz nach; bei dem einen Pferde blieben täglich $32,62 \mathrm{~g}$, bei dem anderen $38,98 \mathrm{~g} \mathrm{~N}$ zurück. Zieht man von diesen $\mathrm{Be}$ trägen im Sinne des oben Gesagten etwa $7 \mathrm{~g}$ als nicht bestimmten $\mathrm{N}$-Verlust $\mathrm{ab}$, so blieben bei Pferd III annühernd $25 \mathrm{~g}$, bei IV $30 \mathrm{~g}$ $\mathrm{N}$ zurück, was 750 resp. $920 \mathrm{~g}$ täglichem Fleischansatz entsprechen würde. Das. Körpergewicht nahm täglich durchschnittlich um 450 resp. $750 \mathrm{~g} \mathrm{zu}$. Es braucht wohl nicht näher erörtert zu werden, dass ebensowenig wie beim $\mathrm{N}$-Deficit auch beim $\mathrm{N}$-Ansatz der berechnete Fleischverlust beziehungsweise Fleischansatz mit der beobachteten Körpergewichtsveränderung nicht übereinstimmen muss resp. nur selten übereinstimmen wird.

Neben dem N-Ansatz liess sich in dieser Versuchsreihe auch ein P-Ansatz nachweisen, bei dem einen Pferde $0,64 \mathrm{~g}$, bei dem anderen $0,53 \mathrm{~g}$. Berechnet man, wie oben auf Grund der $\mathrm{Zu}$ el zerschen Angaben, wie viel P-Ansatz dem N-Ansatz entspricht, so erhält man $0,86 \mathrm{~g}$ resp. $1,1 \mathrm{~g} \mathrm{P}$, also mehr. Als wahrscheinlichste Erklärung dieser Differenz dürfte wohl die Annahme gelten, dass der zurückgebliebene $\mathrm{N}$ ausser zu Fleisch(Muskel)substanz auch noch zur Bildung P-ärmerer Gewebe verwendet wurde, was mit unseren Befunden bei ungenügender Eiweisszufuhr gut übereinstimmt. Bei letzterer werden nämlich, wie oben erwähnt, wahrscheinlich ausser Muskelsubstanz auch P-ärmere Gewebe zersetzt, welch' letztere dagegen bei abundanter Eiweisszufuhr ausser Muskelsubstanz gebildet werden würden.

Die Versuche beweisen jedenfalls, dass zwischen N- und P-Stoffwechsel ein gewisser Parallelismus besteht.

Aus dem Ca- und Mg-Umsatz geht hervor, dass trotz der bedeutenden Ca-Armut des Futters, die resorbirte Ca- und Mg-Menge genügte, den Bedarf zu decken, ja dass von diesen Stoffen sogar noch etwas zurückgehalten wurde. Abgesehen von den Versuchsfehlern ist die thatsächliche Ca-Retention jedenfalls geringer als die gefundene, da einerseits das $\mathrm{Ca}$, das mit den Epidermisgebilden verloren geht, nicht bestimmt wurde und andererseits der etwas grössere oder geringere Staubgehalt des Futters Fehler bei unserer $\mathrm{Ca}-\mathrm{Be}-$ stimmung verursachen konnte. Eben dieser Umstand bedingt eine gewisse Vorsicht in der Beurtheilung der Befunde. Immerhin glaube ich wenigstens im zweiten Versuche auf eine Ca-Retention folgern zu 
können. Weiterhin dürften diese Versuche mit grosser Wahrscheinlichkeit dafür sprechen, dass die im Körper zurückgehaltene Ca-Menge auch beim erwachsenen Thiere der resorbirten Ca-Menge nicht nothwendiger Weise proportional ist. Im ersten Versuche wurden durchschnittlich täglich $15 \mathrm{~g}$ Ca resorbirt, im zweiten Versuche nur $9 \mathrm{~g}$, und doch war die Ca-Retention in diesem Versuche grösser.

Zur Beurtheilung der relativen Grösse der $\mathrm{P}-$, Ca- und $\mathrm{Mg}$ Zufuhr berechnete ich für meine Versuche die täglich pro $1 \mathrm{~kg}$ Körpergewicht verzehrte $\mathrm{P}-$, Ca- und Mg-Menge. Pro Tag und $1 \mathrm{~kg}$ Körpergewicht verzehrten:

\begin{tabular}{|c|c|c|c|c|}
\hline & & $\mathrm{P}$ & $\mathrm{Ca}$ & Mg \\
\hline Pferd III in Versuch. 1 & . & $0,029 \cdot \mathrm{g}$ & $0,090 \mathrm{~g}$ & $0,028, \mathbf{g}$ \\
\hline Pferd IV in Versuch 1 & & $0,027 \mathrm{~g}$ & $0,083 \mathrm{~g}$ & 0,026 \\
\hline ferd III in Versuch 2 & & $0,061 \mathrm{~g}$ & $0,067 \mathrm{~g}$ & 0,037 \\
\hline ferd IV in Versuch 2 & & $0,056 \mathrm{~g}$ & $0,083 \mathrm{~g}$ & 0,084 \\
\hline erd $\mathrm{E}$. & & $0,031 \mathrm{~g}$ & 0,150 & 0,05 \\
\hline
\end{tabular}

Aus solchen Zahlen auf das zur Erhaltung des Gleichgewichtes der fraglichen Elemente im Stoffwechsel nöthige Minimum zu schliessen, hat nur bedingten Werth, denn es kommt ja nicht anf die absolute Menge im verzehrten Futter, sondern auf die Menge des verdaulichen Antheils an. Es wird sich also für jedes Futter ein anderes Minimum ergeben. So viel aber geht aus den mitgetheilten Daten noch hervor, dass bei dem gewöhnlichen gemischten Futter von Pferdeheu und Hafer eine bedeutend geringere Ca-Menge zur Erhaltung des Ca-Gleichgewichts genügt als die Ca-Menge, die in E. Wolff's Versuchen verfüttert wurde. (Führten doch schon $0,069 \mathrm{~g}$ pro $1 \mathrm{~kg}$ und Tag zu einem Ca-Ansatz.) Mit dieser geringen Ca-Menge konnten die Pferde ihr Ca-Gleichgewicht nicht nur während der kurzen Versuchszeit, sondern lange Zeit hindurch erhalten. Wenigstens glaube ich das aus dem Umstande mit einer gewissen Wahrscheinlichkeit folgern zu können, dass sie fast $3 / 4$ Jahr hindurch dieses kalkarme Futter ohne den geringsten Schaden erhielten.

Aus meinem Versuche geht schliesslich auch noch hervor, dass die Menge des Tränkwassers keinen Einfluss hat weder auf die Resorption noch auf den Umsatz des $\mathrm{Ca}$ und Mg. Trotzdem das Pferd III in der ersten Versuchsreihe täglich durchschnittlich um $6,9 \mathrm{l}$, d. h. um $35 \%$ in der Versuchsreihe 2 um $4,7 \mathrm{l}$, d. h. um $27 \%$ mehr Wasser soff als das andere Pferd, war doch der Caund $\mathrm{Mg}$-Umsatz bei beiden Thieren ganz gleich. 